\title{
Minimax-robust filtering problem for stochastic sequences with stationary increments and cointegrated sequences
}

\author{
Maksym Luz $^{1}$ and Mikhail Moklyachuk ${ }^{1, *}$ \\ ${ }^{1}$ Department of Probability Theory, Statistics and Actuarial Mathematics, Taras Shevchenko \\ National University of Kyiv, Ukraine.
}

Received 25 December 2013; Accepted 24 April 2014

Editor: Antanas Zilinskas

\begin{abstract}
The problem of optimal estimation of the linear functionals $A \xi=$ $\sum_{k=0}^{\infty} a(k) \xi(-k)$ and $A_{N} \xi=\sum_{k=0}^{N} a(k) \xi(-k)$ which depend on the unknown values of a stochastic sequence $\xi(k)$ with stationary $n$th increments is considered. Estimates are based on observations of the sequence $\xi(k)+\eta(k)$ at points of time $k=0,-1,-2, \ldots$, where the sequence $\eta(k)$ is stationary and uncorrelated with the sequence $\xi(k)$. Formulas for calculating the mean-square errors and spectral characteristics of the optimal estimates of the functionals are proposed in the case of spectral certainty, where spectral densities of sequences $\xi(k)$ and $\eta(k)$ are exactly known. Minimax (robust) method of estimation is applied in the case where spectral densities of the sequences are not known exactly, but sets of admissible spectral densities are given. Formulas that determine the least favorable spectral densities and minimax spectral characteristics are proposed for some special classes of admissible densities. The filtering problem for a class of cointegrated sequences is investigated.
\end{abstract}

Keywords Stochastic sequence with stationary increments; cointegrated sequence; minimax-robust estimate; mean square error; least favorable spectral density; minimax-robust spectral characteristic

DOI: $10.19139 /$ soic.v2i3.56

\footnotetext{
${ }^{*}$ Correspondence to: Department of Probability Theory, Statistics and Actuarial Mathematics, Taras Shevchenko National University of Kyiv, Kyiv 01601, Ukraine. E-mail: moklyachuk@gmail.com
}

ISSN 2310-5070 (online) ISSN 2311-004X (print)

Copyright (C) 2014 International Academic Press 


\section{Introduction}

Wide sense stationary and related stochastic processes have been studied by many scientists. The obtained results found their applications for solving actual problems in description and analysis of models of economic and financial time series. The most simple examples are linear stationary models such as moving average (MA), autoregressive (AR) or autoregressive-moving average (ARMA) sequences, state space model, all of which refer to stationary sequences with rational spectral function without unit AR-roots. Time series with trends and seasonal components are modeled by integrated ARMA (ARIMA) sequences which have unit roots in their autoregressive parts and are examples of sequences with stationary increments. Such models attract interest of scientists during the last 30 years. The main points concerning model definition, parameter estimation, forecasting and further investigation are discussed in the well-known book by Box, Jenkins and Reinsel [1]. While analyzing financial data some economists noticed that in special cases linear combinations of integrated sequences become stationary. Grander [10] called this phenomenon cointegration. Cointegrated sequences found their application in applied and theoretical econometrics and financial time series analysis [9].

Estimation of unknown values of stochastic processes is an important part of the theory of stochastic processes. Effective methods of solution of the linear extrapolation, interpolation and filtering problems for stationary stochastic processes were developed by Kolmogorov [15], Wiener [37], Yaglom [38, 39]. Further results one can find in book by Rozanov [35]. Yaglom [40, 41] developed theory of non-stationary processes whose increments of order $n$ form a stationary process. Further results for such stochastic processes were presented by Pinsker [33], Yaglom and Pinsker [32]. See books by Yaglom [38, 39] for more relative results and references.

The mean square optimal estimation problems for stochastic processes with $n$th stationary increments are natural generalizations of extrapolation, interpolation and filtering problems for stationary stochastic processes. The classical methods of solution of extrapolation, interpolation and filtering problems are based on the assumption that spectral density of the process is known. In practice, however, it is impossible to obtain complete information on the spectral density in most cases. To solve the problem one finds parametric or nonparametric estimates of the unknown spectral density or selects a density by other reasoning. Then the classical estimation method is applied provided that the estimated or selected density is the true one. Vastola and Poor [36] have demonstrated that the described procedure can result in significant increasing of the value of error. This is a reason for searching estimates which are optimal for all densities from a certain class of admissible spectral densities. These estimates are called minimax since they minimize the maximal value of the error of estimates. A survey of results 
in minimax (robust) methods of data processing can be found in the paper by Kassam and Poor [14]. The paper by Grenander [11] should be marked as the first one where the minimax extrapolation problem for stationary processes was formulated and solved. Franke and Poor [13], Franke [12] investigated the minimax extrapolation and filtering problems for stationary sequences with the help of convex optimization methods. In papers by Moklyachuk [20] - [22] the minimax approach was applied to extrapolation, interpolation and filtering problems for functionals which depend on the unknown values of stationary processes and sequences. For more results and details see, for example, book by Moklyachuk [29], articles and book by Moklyachuk and Masyutka [26] - [31]. Dubovets'ka and Moklyachuk [3] - [8] investigated the minimax-robust estimation problems (extrapolation, interpolation and filtering) for periodically correlated stochastic processes.

In papers [16] - [19], [25] by Luz and Moklyachuk the minimax interpolation and extrapolation problems for linear functionals which depend on unknown (missed) values of stochastic process $\xi(m)$ with stationary $n$th increments from observations of the process with and without noise were investigated. In the paper [18] they investigated the problem of optimal linear estimation of the functional $A \xi=\sum_{k=0}^{\infty} a(k) \xi(-k)$ which depends on unknown values of a stochastic sequence $\xi(k)$ with $n$th stationary increments from observations of the sequence $\xi(k)+\eta(k)$ at points $k=0,-1,-2, \ldots$, where $\eta(k)$ is a stochastic sequence with stationary $n$th increments which is uncorrelated with the sequence $\xi(k)$. To solve the problem the functional $A \xi$ is represented as a functional of increments of the sequence providing some conditions on the coefficients $a(k), k \geq 0$. This representation gives a possibility to find an estimate only for the functional $A \xi$, but not for the functional $A_{N} \xi=\sum_{k=0}^{N} a(k) \xi(-k)$. In the present article we require the noise sequence $\eta(k)$ to be stationary. This condition let us find estimates of both functionals $A \eta=\sum_{k=0}^{\infty} a(k) \eta(-k)$ and $A_{N} \eta=\sum_{k=0}^{N} a(k) \eta(-k)$ which are used for solving the filtering problem for functionals $A \xi$ and $A_{N} \xi$. The obtained results give us a method of solution the filtering problem for cointegrated sequences $\xi(k)$ and $\zeta(k)$ under the condition that a stationary linear combination of the sequences does not correlate with the sequence $\xi(k)$. The estimation problem is solved in the case of spectral certainty where spectral densities of sequences $\xi(k)$ and $\eta(k)$ are exactly known as well as in the case of spectral uncertainty where spectral densities of the sequences are not exactly known, but a set of admissible spectral densities is given. Formulas that determine least favorable spectral densities and minimax (robust) spectral characteristics of optimal estimates of functionals are proposed in the case of spectral uncertainty for concrete classes of admissible spectral densities. 


\section{Stationary increment stochastic sequences. Spectral representation}

\section{Definition 2.1}

For a given stochastic sequence $\{\xi(m), m \in \mathbb{Z}\}$ the sequence

$$
\xi^{(n)}(m, \mu)=\left(1-B_{\mu}\right)^{n} \xi(m)=\sum_{l=0}^{n}(-1)^{l} C_{n}^{l} \xi(m-l \mu),
$$

where $B_{\mu}$ is a backward shift operator with step $\mu \in \mathbb{Z}$, such that $B_{\mu} \xi(m)=$ $\xi(m-\mu)$, is called stochastic $n$th increment sequence with step $\mu \in \mathbb{Z}$.

For the stochastic $n$th increment sequence $\xi^{(n)}(m, \mu)$ the following relations hold true

$$
\begin{gathered}
\xi^{(n)}(m,-\mu)=(-1)^{n} \xi^{(n)}(m+n \mu, \mu), \\
\xi^{(n)}(m, k \mu)=\sum_{l=0}^{(k-1) n} A_{l} \xi^{(n)}(m-l \mu, \mu), \quad k \in \mathbb{N},
\end{gathered}
$$

where coefficients $\left\{A_{l}, l=0,1,2, \ldots,(k-1) n\right\}$ are determined by the representation

$$
\left(1+x+\ldots+x^{k-1}\right)^{n}=\sum_{l=0}^{(k-1) n} A_{l} x^{l}
$$

\section{Definition 2.2}

The stochastic $n$th increment sequence $\xi^{(n)}(m, \mu)$ generated by stochastic sequence $\{\xi(m), m \in \mathbb{Z}\}$ is wide sense stationary if the mathematical expectations

$$
\begin{gathered}
\mathrm{E} \xi^{(n)}\left(m_{0}, \mu\right)=c^{(n)}(\mu), \\
\mathrm{E} \xi^{(n)}\left(m_{0}+m, \mu_{1}\right) \xi^{(n)}\left(m_{0}, \mu_{2}\right)=D^{(n)}\left(m, \mu_{1}, \mu_{2}\right)
\end{gathered}
$$

exist for all $m_{0}, \mu, m, \mu_{1}, \mu_{2}$ and do not depend on $m_{0}$. The function $c^{(n)}(\mu)$ is called mean value of the $n$th increment sequence and the function $D^{(n)}\left(m, \mu_{1}, \mu_{2}\right)$ is called structural function of the stationary $n$th increment sequence (or structural function of $n$th order of the stochastic sequence $\{\xi(m), m \in \mathbb{Z}\})$.

The stochastic sequence $\{\xi(m), m \in \mathbb{Z}\}$ which determines the stationary $n$th increment sequence $\xi^{(n)}(m, \mu)$ by formula (1) is called sequence with stationary $n$th increments (or integrated sequence of order $n$ ).

\section{Theorem 2.1}

The mean value $c^{(n)}(\mu)$ and the structural function $D^{(n)}\left(m, \mu_{1}, \mu_{2}\right)$ of the stochastic stationary $n$th increment sequence $\xi^{(n)}(m, \mu)$ can be represented in the following forms

$$
\begin{gathered}
c^{(n)}(\mu)=c \mu^{n}, \\
D^{(n)}\left(m, \mu_{1}, \mu_{2}\right)=\int_{-\pi}^{\pi} e^{i \lambda m}\left(1-e^{-i \mu_{1} \lambda}\right)^{n}\left(1-e^{i \mu_{2} \lambda}\right)^{n} \frac{1}{\lambda^{2 n}} d F(\lambda),
\end{gathered}
$$


where $c$ is a constant, $F(\lambda)$ is a left-continuous nondecreasing bounded function with $F(-\pi)=0$. The constant $c$ and the function $F(\lambda)$ are determined uniquely by the increment sequence $\xi^{(n)}(m, \mu)$.

From the other hand, a function $c^{(n)}(\mu)$ which has form (4) with a constant $c$ and a function $D^{(n)}\left(m, \mu_{1}, \mu_{2}\right)$ which has form (5) with a function $F(\lambda)$ which satisfies the indicated conditions are the mean value and the structural function of a stationary $n$th increment sequence $\xi^{(n)}(m, \mu)$.

Using representation (5) of the structural function of a stationary $n$th increment sequence $\xi^{(n)}(m, \mu)$ and the Karhunen theorem [2], we obtain the following spectral representation of the stationary $n$th increment sequence $\xi^{(n)}(m, \mu)$ :

$$
\xi^{(n)}(m, \mu)=\int_{-\pi}^{\pi} e^{i m \lambda}\left(1-e^{-i \mu \lambda}\right)^{n} \frac{1}{(i \lambda)^{n}} d Z(\lambda),
$$

where $Z(\lambda)$ is an orthogonal stochastic measure on $[-\pi, \pi)$ connected with the spectral function $F(\lambda)$ by the relation

$$
\mathrm{E} Z\left(A_{1}\right) \overline{Z\left(A_{2}\right)}=F\left(A_{1} \cap A_{2}\right)<\infty .
$$

\section{Filtering problem}

Let a stochastic sequence $\{\xi(m), m \in \mathbb{Z}\}$ define stationary $n$th increment sequence $\xi^{(n)}(m, \mu)$ with absolutely continuous spectral function $F(\lambda)$ which has spectral density $f(\lambda)$. Let $\{\eta(m), m \in \mathbb{Z}\}$ be an uncorrelated with the sequence $\xi(m)$ stationary stochastic sequence with absolutely continuous spectral function $G(\lambda)$ which has spectral density $g(\lambda)$. Without loss of generality we will assume that mean values of the increment sequence $\xi^{(n)}(m, \mu)$ and stationary sequence $\eta(m)$ equal to 0 .

Consider the problem of mean-square optimal linear estimation of the functionals

$$
A \xi=\sum_{k=0}^{\infty} a(k) \xi(-k), \quad A_{N} \xi=\sum_{k=0}^{N} a(k) \xi(-k)
$$

which depend on unknown values of the sequence $\xi(m)$ from observations of the sequence $\zeta(m)=\xi(m)+\eta(m)$ at points $m=0,-1,-2, \ldots$ We will consider the case where the step $\mu>0$. And we will suppose that conditions

$$
\sum_{k=0}^{\infty}\left|a_{\mu}(k-\mu n)\right|<\infty, \quad \sum_{k=0}^{\infty}(k+1)\left|a_{\mu}(k-\mu n)\right|^{2}<\infty
$$

hold true for coefficients $a_{\mu}(k), k \geq-\mu n$, which are defined in the following part of the paper. 
The functional $A \xi$ can be represented in the form

$$
A \xi=A \zeta-A \eta
$$

where $A \zeta=\sum_{k=0}^{\infty} a(k) \zeta(-k), A \eta=\sum_{k=0}^{\infty} a(k) \eta(-k)$.

Let $\Delta(f, g, \widehat{A} \xi)=\mathrm{E}|A \xi-\widehat{A} \xi|^{2}$ denote the mean-square error of the estimate $\widehat{A} \xi$ of the functional $A \xi$ and let $\Delta(f, g, \widehat{A} \eta)=\mathrm{E}|A \eta-\widehat{A} \eta|^{2}$ denote the meansquare error of the estimate $\widehat{A} \eta$ of the functional $A \eta$. Since the functional $A \zeta$ is determined by the observed values of the sequence $\zeta(m)$, the following relations hold true

$$
\widehat{A} \xi=A \zeta-\widehat{A} \eta
$$

$\Delta(f, g, \widehat{A} \xi)=\mathrm{E}|A \xi-\widehat{A} \xi|^{2}=\mathrm{E}|A \zeta-A \eta-A \zeta+\widehat{A} \eta|^{2}=\mathrm{E}|A \eta-\widehat{A} \eta|^{2}=\Delta(f, g, \widehat{A} \eta)$.

To find the mean-square optimal estimate of the functional $A \eta$ we use spectral representations of the stationary sequence $\eta(m)$, the stationary $n$th increment sequence $\eta^{(n)}(m, \mu)$ and apply the Hilbert space orthogonal projection method proposed by Kolmogorov [15]. The stationary stochastic sequence $\eta(m)$ admits the spectral representation

$$
\eta(m)=\int_{-\pi}^{\pi} e^{i \lambda m} d Z_{\eta}(\lambda)
$$

and the $n$th increment sequence $\eta^{(n)}(m, \mu)$ admits the spectral representation

$$
\eta^{(n)}(m, \mu)=\int_{-\pi}^{\pi} e^{i \lambda m}\left(1-e^{-i \lambda \mu}\right)^{n} d Z_{\eta}(\lambda),
$$

where $Z_{\eta}(\lambda)$ is an orthogonal stochastic measure on $[-\pi, \pi)$ corresponding to the spectral function $G(\lambda)$. The stationary increment sequence $\zeta^{(n)}(m, \mu)$ admits the spectral representation

$$
\begin{gathered}
\zeta^{(n)}(m, \mu)=\int_{-\pi}^{\pi} e^{i \lambda m}\left(1-e^{-i \lambda \mu}\right)^{n} \frac{1}{(i \lambda)^{n}} d Z_{\xi^{(n)}+\eta^{(n)}}(\lambda) \\
=\int_{-\pi}^{\pi} e^{i \lambda m}\left(1-e^{-i \lambda \mu}\right)^{n} \frac{1}{(i \lambda)^{n}} d Z_{\xi^{(n)}}(\lambda)+\int_{-\pi}^{\pi} e^{i \lambda m}\left(1-e^{-i \lambda \mu}\right)^{n} d Z_{\eta}(\lambda),
\end{gathered}
$$

where $d Z_{\eta^{(n)}}(\lambda)=(i \lambda)^{n} d Z_{\eta}(\lambda), \lambda \in[-\pi, \pi)$. The spectral density $p(\lambda)$ of the sequence $\zeta(m)$ is determined by spectral densities $f(\lambda)$ and $g(\lambda)$ by the relation

$$
p(\lambda)=f(\lambda)+\lambda^{2 n} g(\lambda)
$$


Denote by $H^{0}\left(\xi_{\mu}^{(n)}+\eta_{\mu}^{(n)}\right)$ the closed linear subspace of the Hilbert space $H=L_{2}(\Omega, \mathfrak{F}, \mathrm{P})$ of random variables of the second order which is generated by values $\left\{\xi^{(n)}(k, \mu)+\eta^{(n)}(k, \mu): k \leq 0\right\}, \mu>0$.

Denote by $L_{2}^{0}\left(f(\lambda)+\lambda^{2 n} g(\lambda)\right)$ the closed linear subspace of the Hilbert space $L_{2}\left(f(\lambda)+\lambda^{2 n} g(\lambda)\right)$ generated by functions

$$
\left\{e^{i \lambda k}\left(1-e^{-i \lambda \mu}\right)^{n} \frac{1}{(i \lambda)^{n}}: k \leq 0\right\} .
$$

It follows from the formula

$$
\xi^{(n)}(k, \mu)+\eta^{(n)}(k, \mu)=\int_{-\pi}^{\pi} e^{i \lambda k}\left(1-e^{-i \lambda \mu}\right)^{n} \frac{1}{(i \lambda)^{n}} d Z_{\xi^{(n)}+\eta^{(n)}}(\lambda)
$$

that there exists a one to one correspondence between elements $e^{i \lambda k}(1-$ $\left.e^{-i \lambda \mu}\right)^{n} \frac{1}{(i \lambda)^{n}}$ of the space $L_{2}^{0}\left(f(\lambda)+\lambda^{2 n} g(\lambda)\right)$ and elements $\xi^{(n)}(k, \mu)+$ $\eta^{(n)}(k, \mu)$ of the space $H^{0}\left(\xi_{\mu}^{(n)}+\eta_{\mu}^{(n)}\right)$.

Every linear estimate $\widehat{A} \xi$ of the functional $A \xi$ admits the representation

$$
\widehat{A} \xi=A \zeta-\int_{-\pi}^{\pi} h_{\mu}(\lambda) d Z_{\xi^{(n)}+\eta^{(n)}}(\lambda),
$$

where $h_{\mu}(\lambda)$ is the spectral characteristic of the estimate $\widehat{A} \eta$. The mean square optimal estimate $\widehat{A} \eta$ is a projection of the element $A \eta$ on the subspace $H^{0}\left(\xi_{\mu}^{(n)}+\right.$ $\left.\eta_{\mu}^{(n)}\right)$. This estimate is determined by the following conditions:

1) $\widehat{A} \eta \in H^{0}\left(\xi_{\mu}^{(n)}+\eta_{\mu}^{(n)}\right)$;

2) $(A \eta-\widehat{A} \eta) \perp H^{0}\left(\xi_{\mu}^{(n)}+\eta_{\mu}^{(n)}\right)$.

It comes from condition 2) that for all $k \leq 0$ the following relations hold true

$$
\begin{gathered}
\mathrm{E}(A \eta-\widehat{A} \eta)\left(\overline{\xi^{(n)}(k, \mu)+\eta^{(n)}(k, \mu)}\right) \\
=\frac{1}{2 \pi} \int_{-\pi}^{\pi}\left(A\left(e^{-i \lambda}\right)-(i \lambda)^{n} h_{\mu}(\lambda)\right) e^{-i \lambda k}\left(1-e^{i \lambda \mu}\right)^{n} g(\lambda) d \lambda \\
-\frac{1}{2 \pi} \int_{-\pi}^{\pi} h_{\mu}(\lambda) e^{-i \lambda k}\left(1-e^{i \lambda \mu}\right)^{n} \frac{1}{(-i \lambda)^{n}} f(\lambda) d \lambda=0 .
\end{gathered}
$$

These relations can be represented in the form

$$
\begin{gathered}
\int_{-\pi}^{\pi}\left[A\left(e^{-i \lambda}\right) g(\lambda)(-i \lambda)^{n}-h_{\mu}(\lambda)\left(f(\lambda)+\lambda^{2 n} g(\lambda)\right)\right] \frac{\left(1-e^{i \lambda \mu}\right)^{n}}{(-i \lambda)^{n}} e^{-i \lambda k} d \lambda \\
=0, k \leq 0 .
\end{gathered}
$$

Stat., Optim. Inf. Comput. Vol. 2, September 2014. 
From these equations we can derive the spectral characteristic $h_{\mu}(\lambda)$ of the estimate $\widehat{A} \eta$. It is of the form

$$
\begin{gathered}
h_{\mu}(\lambda)=A\left(e^{-i \lambda}\right) \frac{(-i \lambda)^{n} g(\lambda)}{f(\lambda)+\lambda^{2 n} g(\lambda)}-\frac{(-i \lambda)^{n} C_{\mu}\left(e^{i \lambda}\right)}{\left(1-e^{i \lambda \mu}\right)^{n}\left(f(\lambda)+\lambda^{2 n} g(\lambda)\right)}, \\
A\left(e^{-i \lambda}\right)=\sum_{k=0}^{\infty} a(k) e^{-i \lambda k}, \quad C_{\mu}\left(e^{i \lambda}\right)=\sum_{k=1}^{\infty} c_{\mu}(k) e^{i \lambda k} .
\end{gathered}
$$

where $c_{\mu}(k)$ are unknown coefficients to be determined. It follows from condition 1) that the spectral characteristic $h_{\mu}(\lambda)$ of the estimate $\widehat{A} \eta$ admits the representation

$$
h_{\mu}(\lambda)=h(\lambda)\left(1-e^{-i \lambda \mu}\right)^{n} \frac{1}{(i \lambda)^{n}}, \quad h(\lambda)=\sum_{k=0}^{\infty} s(k) e^{-i \lambda k}
$$

where

$$
\begin{gathered}
\int_{-\pi}^{\pi}|h(\lambda)|^{2}\left|1-e^{i \lambda \mu}\right|^{2 n} \frac{f(\lambda)+\lambda^{2 n} g(\lambda)}{\lambda^{2 n}} d \lambda<\infty \\
\frac{(i \lambda)^{n} h_{\mu}(\lambda)}{\left(1-e^{-i \lambda \mu}\right)^{n}} \in L_{2}^{0}, \\
\int_{-\pi}^{\pi}\left[\frac{A\left(e^{-i \lambda}\right) \lambda^{2 n} g(\lambda)}{\left(1-e^{-i \lambda \mu}\right)^{n}\left(f(\lambda)+\lambda^{2 n} g(\lambda)\right)}-\frac{\lambda^{2 n} C_{\mu}\left(e^{i \lambda}\right)}{\left|1-e^{i \lambda \mu}\right|^{2 n}\left(f(\lambda)+\lambda^{2 n} g(\lambda)\right)}\right] e^{-i \lambda l} d \lambda=0, l \geq 1 .
\end{gathered}
$$

Suppose that spectral densities $f(\lambda), g(\lambda)$ are such that the following condition holds true:

$$
\int_{-\pi}^{\pi} \frac{\lambda^{2 n}}{\left|1-e^{i \lambda \mu}\right|^{2 n}\left(f(\lambda)+\lambda^{2 n} g(\lambda)\right)} d \lambda<\infty .
$$

Determine for every $k, j \in \mathbb{Z}$ the Fourier coefficients of the corresponding functions

$$
\begin{gathered}
S_{k, j}^{\mu}=\frac{1}{2 \pi} \int_{-\pi}^{\pi} e^{-i \lambda(j+k)} \frac{\lambda^{2 n} g(\lambda)}{\left|1-e^{i \lambda \mu}\right|^{2 n}\left(f(\lambda)+\lambda^{2 n} g(\lambda)\right)} d \lambda \\
P_{k, j}^{\mu}=\frac{1}{2 \pi} \int_{-\pi}^{\pi} e^{i \lambda(j-k)} \frac{\lambda^{2 n}}{\mid 1-e^{\left.i \lambda \mu\right|^{2 n}\left(f(\lambda)+\lambda^{2 n} g(\lambda)\right)} d \lambda} \\
Q_{k, j}=\frac{1}{2 \pi} \int_{-\pi}^{\pi} e^{i \lambda(j-k)} \frac{f(\lambda) g(\lambda)}{\left(f(\lambda)+\lambda^{2 n} g(\lambda)\right)} d \lambda
\end{gathered}
$$

Using these Fourier coefficients we can represent equation (11) in the form of the following system of linear equations

$$
\sum_{m=-\mu n}^{\infty} S_{l, m}^{\mu} a_{\mu}(m)=\sum_{k=1}^{\infty} P_{l, k}^{\mu} c_{\mu}(k), \quad l \geq 1,
$$

Stat., Optim. Inf. Comput. Vol. 2, September 2014. 
where

$$
a_{\mu}(m)=\sum_{l=\max \left\{\left[-\frac{m}{\mu}\right]^{\prime}, 0\right\}}^{n}(-1)^{l} C_{n}^{l} a(m+\mu l), \quad m \geq-\mu n,
$$

Here $[x]^{\prime}$ denotes the least integer number among numbers which are greater or equal to $x$. This system can be written in the form

$$
\mathbf{S}_{\mu} \mathbf{a}_{\mu}=\mathbf{P}_{\mu} \mathbf{c}_{\mu},
$$

where $\mathbf{c}_{\mu}=\left(c_{\mu}(1), c_{\mu}(2), c_{\mu}(2), \ldots\right)^{\prime}, \mathbf{a}_{\mu}=\left(a_{\mu}(-\mu n), a_{\mu}(-(\mu n-1)), \ldots\right)^{\prime}, \mathbf{P}_{\mu}$, $\mathbf{S}_{\mu}$ are linear operators in the space $\ell_{2}$ defined by the matrices with elements $\left(\mathbf{P}_{\mu}\right)_{l, k}=P_{l, k}^{\mu}, l, k \geq 1,\left(\mathbf{S}_{\mu}\right)_{l, m}=S_{l, m}^{\mu}, l \geq 1, m \geq-\mu n$. Consequently, the unknown coefficients $c_{\mu}(k)$ which determine the spectral characteristic $h_{\mu}(\lambda)$ are calculated by the formula

$$
c_{\mu}(k)=\left(\mathbf{P}_{\mu}^{-1} \mathbf{S}_{\mu} \mathbf{a}_{\mu}\right)_{k}, \quad k \geq 0,
$$

where $\left(\mathbf{P}_{\mu}^{-1} \mathbf{S}_{\mu} \mathbf{a}_{\mu}\right)_{k}, k \geq 0$, is the $k$ th element of the vector $\mathbf{P}_{\mu}^{-1} \mathbf{S}_{\mu} \mathbf{a}_{\mu}$. Thus, the spectral characteristic $h_{\mu}(\lambda)$ of the optimal estimate $\widehat{A} \eta$ of the functional $A \eta$ is calculated by the formula

$$
h_{\mu}(\lambda)=A\left(e^{-i \lambda}\right) \frac{(-i \lambda)^{n} g(\lambda)}{f(\lambda)+\lambda^{2 n} g(\lambda)}-\frac{(-i \lambda)^{n} \sum_{k=1}^{\infty}\left(\mathbf{P}_{\mu}^{-1} \mathbf{S}_{\mu} \mathbf{a}_{\mu}\right)_{k} e^{i \lambda k}}{\left(1-e^{i \lambda \mu}\right)^{n}\left(f(\lambda)+\lambda^{2 n} g(\lambda)\right)} .
$$

The mean-square error of the estimate is calculated by the formula

$$
\begin{gathered}
\Delta(f, g ; \widehat{A} \xi)=\Delta(f, g ; \widehat{A} \eta)=\mathrm{E}|A \eta-\widehat{A} \eta|^{2} \\
=\frac{1}{2 \pi} \int_{-\pi}^{\pi} \frac{\left|A\left(e^{-i \lambda}\right)\left(1-e^{i \lambda \mu}\right)^{n} f(\lambda)+\lambda^{2 n} \sum_{k=1}^{\infty}\left(\mathbf{P}_{\mu}^{-1} \mathbf{S}_{\mu} \mathbf{a}_{\mu}\right)_{k} e^{i \lambda k}\right|^{2}}{\left|1-e^{i \lambda \mu}\right|^{2 n}\left(f(\lambda)+\lambda^{2 n} g(\lambda)\right)^{2}} g(\lambda) d \lambda \\
+\frac{1}{2 \pi} \int_{-\pi}^{\pi} \frac{\left|A\left(e^{-i \lambda}\right)\left(1-e^{i \lambda \mu}\right)^{n} \lambda^{2 n} g(\lambda)-\lambda^{2 n} \sum_{k=1}^{\infty}\left(\mathbf{P}_{\mu}^{-1} \mathbf{S}_{\mu} \mathbf{a}_{\mu}\right)_{k} e^{i \lambda k}\right|^{2}}{\lambda^{2 n}\left|1-e^{i \lambda \mu}\right|^{2 n}\left(f(\lambda)+\lambda^{2 n} g(\lambda)\right)^{2}} f(\lambda) d \lambda \\
=\left\langle\mathbf{S}_{\mu} \mathbf{a}_{\mu}, \mathbf{P}_{\mu}^{-1} \mathbf{S}_{\mu} \mathbf{a}_{\mu}\right\rangle+\langle\mathbf{Q} \mathbf{a}, \mathbf{a}\rangle,
\end{gathered}
$$

where $\mathbf{a}=(a(0), a(1), a(2), \ldots)^{\prime}, \mathbf{Q}$ is a linear operator in the space $\ell_{2}$ defined by the matrix with elements $(\mathbf{Q})_{l, k}=Q_{l, k}, l, k \geq 0$.

These observations can be summarized in the form of the theorem.

\section{Theorem 3.1}

Let $\{\xi(m), m \in \mathbb{Z}\}$ be a stochastic sequence which defines stationary $n$th increment sequence $\xi^{(n)}(m, \mu)$ with absolutely continuous spectral function $F(\lambda)$ which has spectral density $f(\lambda)$. Let $\{\eta(m), m \in \mathbb{Z}\}$ be an uncorrelated with the sequence $\xi(m)$ stationary stochastic sequence with absolutely continuous spectral 
function $G(\lambda)$ which has spectral density $g(\lambda)$. Let condition (12) be satisfied. Let coefficients $\left\{a_{\mu}(k): k \geq-\mu n\right\}$ defined by formula (13) satisfy conditions (8). The optimal linear estimate $\widehat{A} \xi$ of the functional $A \xi$ of unknown elements $\xi(m), m \leq 0$, based on observations of the sequence $\xi(m)+\eta(m)$ at points $m=0,-1,-2, \ldots$ is calculated by formula (10). The spectral characteristic $h_{\mu}(\lambda)$ of the optimal estimate $\widehat{A} \xi$ is calculated by formula (14). The value of the meansquare error $\Delta(f, g ; \widehat{A} \xi)$ is calculated by formula $(15)$.

We can use Theorem 3.1 to obtain the optimal estimate $\widehat{A}_{N} \xi$ of the functional $A_{N} \xi$ of unknown elements $\xi(m), m=0,-1,-2, \ldots,-N$, from observations of the sequence $\xi(m)+\eta(m)$ at points $m=0,-1,-2, \ldots$. Take $a(k)=0, k>N$. Then the spectral characteristic $h_{\mu, N}(\lambda)$ of the linear estimate

$$
\widehat{A}_{N} \xi=A_{N} \zeta-\int_{-\pi}^{\pi} h_{\mu, N}(\lambda) d Z_{\xi^{(n)}+\eta^{(n)}}(\lambda)
$$

is calculated by the formula

$$
h_{\mu, N}(\lambda)=A_{N}\left(e^{-i \lambda}\right) \frac{(-i \lambda)^{n} g(\lambda)}{f(\lambda)+\lambda^{2 n} g(\lambda)}-\frac{(-i \lambda)^{n} \sum_{k=1}^{\infty}\left(\mathbf{P}_{\mu}^{-1} \mathbf{S}_{\mu, N} \mathbf{a}_{\mu, N}\right)_{k} e^{i \lambda k}}{\left(1-e^{i \lambda \mu}\right)^{n}\left(f(\lambda)+\lambda^{2 n} g(\lambda)\right)}
$$

where

$$
\begin{gathered}
A_{N}\left(e^{-i \lambda}\right)=\sum_{k=0}^{N} a(k) e^{-i \lambda k}, \\
\mathbf{a}_{\mu, N}=\left(a_{\mu, N}(-\mu n), a_{\mu, N}(-(\mu n-1)), \ldots, a_{\mu, N}(N), 0, \ldots\right), \\
a_{\mu, N}(m)=\sum_{l=\max \left\{\left[-\frac{m}{\mu}\right]^{\prime}, 0\right\}}^{\min \left\{\left[\frac{N-m}{\mu}\right], n\right\}}(-1)^{l} C_{n}^{l} a(m+\mu l), \quad-\mu n \leq m \leq N,
\end{gathered}
$$

$\mathbf{S}_{\mu, N}$ is a linear operator in the space $\ell_{2}$ defined by the matrix with elements $\left(\mathbf{S}_{\mu, N}\right)_{l, m}=S_{l, m}^{\mu}, l \geq 1,-\mu n \leq m \leq N$, and $\left(\mathbf{S}_{\mu, N}\right)_{l, m}=0, l \geq 1, m>N$. The mean-square error of the estimate $\widehat{A}_{N} \xi$ is calculated by the formula

$$
\begin{gathered}
\Delta\left(f, g ; \widehat{A}_{N} \xi\right)=\Delta\left(f, g ; \widehat{A}_{N} \eta\right)=\mathrm{E}\left|A_{N} \eta-\widehat{A}_{N} \eta\right|^{2} \\
=\frac{1}{2 \pi} \int_{-\pi}^{\pi} \frac{\left|A_{N}\left(e^{-i \lambda}\right)\left(1-e^{i \lambda \mu}\right)^{n} f(\lambda)+\lambda^{2 n} \sum_{k=1}^{\infty}\left(\mathbf{P}_{\mu}^{-1} \mathbf{S}_{\mu, N} \mathbf{a}_{\mu, N}\right)_{k} e^{i \lambda k}\right|^{2}}{\left|1-e^{i \lambda \mu}\right|^{2 n}\left(f(\lambda)+\lambda^{2 n} g(\lambda)\right)^{2}} g(\lambda) d \lambda \\
+\frac{1}{2 \pi} \int_{-\pi}^{\pi} \frac{\left|A_{N}\left(e^{-i \lambda}\right)\left(1-e^{i \lambda \mu}\right)^{n} \lambda^{2 n} g(\lambda)-\lambda^{2 n} \sum_{k=1}^{\infty}\left(\mathbf{P}_{\mu}^{-1} \mathbf{S}_{\mu, N} \mathbf{a}_{\mu, N}\right)_{k} e^{i \lambda k}\right|^{2}}{\lambda^{2 n}\left|1-e^{i \lambda \mu}\right|^{2 n}\left(f(\lambda)+\lambda^{2 n} g(\lambda)\right)^{2}} f(\lambda) d \lambda \\
=\left\langle\mathbf{S}_{\mu, N} \mathbf{a}_{\mu, N}, \mathbf{P}_{\mu}^{-1} \mathbf{S}_{\mu, N} \mathbf{a}_{\mu, N}\right\rangle+\left\langle\mathbf{Q}_{N} \mathbf{a}_{N}, \mathbf{a}_{N}\right\rangle
\end{gathered}
$$


where $\mathbf{a}_{N}=(a(0), a(1), \ldots, a(N), 0, \ldots), \mathbf{Q}_{N}$ is a linear operator in the space $\ell_{2}$ defined by the matrix with elements $\left(\mathbf{Q}_{N}\right)_{l, k}=Q_{l, k}^{\mu}, 0 \leq l, k \leq N$, and $\left(\mathbf{Q}_{N}\right)_{l, k}=0$ otherwise.

The following theorem holds true.

\section{Theorem 3.2}

Let $\{\xi(m), m \in \mathbb{Z}\}$ be a stochastic sequence which defines stationary $n$th increment sequence $\xi^{(n)}(m, \mu)$ with an absolutely continuous spectral function $F(\lambda)$ which has spectral density $f(\lambda)$. Let $\{\eta(m), m \in \mathbb{Z}\}$ be an uncorrelated with the sequence $\xi(m)$ stationary stochastic sequence with an absolutely continuous spectral function $G(\lambda)$ which has spectral density $g(\lambda)$. Let condition (12) be satisfied. Let coefficients $\left\{a_{\mu, N}(k):-\mu n \leq k \leq N\right\}$ be defined by formula (18). The optimal linear estimate $\widehat{A}_{N} \xi$ of the functional $A_{N} \xi$ of unknown elements $\xi(k), k=0,-1,-2, \ldots,-N$, from observations of the sequence $\xi(m)+\eta(m)$ at points $m=0,-1,-2, \ldots$ is calculated by formula (16). The spectral characteristic $h_{\mu, N}(\lambda)$ of the optimal estimate $\widehat{A}_{N} \xi$ is calculated by formula (17). The value of the mean-square error $\Delta\left(f, g ; \widehat{A}_{N} \xi\right)$ is calculated by formula (19).

A particular case of the considered problem is the problem of estimation of an unobserved value $\xi(-p)$ at a point $-p, p \geq 0$, from observations of the sequence $\xi(k)+\eta(k)$ at points $k=0,-1,-2, \ldots$. In this case the vector $\mathbf{a}_{\mu, N}$ has coefficients $a_{\mu, N}(m)=(-1)^{l} C_{n}^{l}$ if $m=p-\mu l, l=$ $0,1,2, \ldots, n, \quad m=-\mu n,-(\mu n-1), \ldots, \quad$ and $a_{\mu, N}(m)=0$ if $m \neq p-\mu l$, $l=0,1,2, \ldots, n, \quad m=-\mu n,-(\mu n-1), \ldots$. Let us define a vector $\mathbf{a}_{n}=$ $\left(a_{n}(0), a_{n}(1), \ldots, a_{n}(n), 0,0, \ldots\right)^{\prime}$, where $a_{n}(k)=(-1)^{k} C_{n}^{k}, k=0,1,2, \ldots, n$. It follows from the derived formulas that the spectral characteristic $h_{\mu, p}(\lambda)$ of the optimal estimate

$$
\widehat{\xi}(-p)=\zeta(-p)-\int_{-\pi}^{\pi} h_{\mu, p}(\lambda) d Z_{\xi^{(n)}+\eta^{(n)}}(\lambda)
$$

of the value $\xi(-p), p \geq 0$, can be calculated by the formula

$$
h_{\mu, p}(\lambda)=e^{-i \lambda p} \frac{(-i \lambda)^{n} g(\lambda)}{f(\lambda)+\lambda^{2 n} g(\lambda)}-\frac{(-i \lambda)^{n} \sum_{k=1}^{\infty}\left(\mathbf{P}_{\mu}^{-1} \mathbf{S}_{\mu, p} \mathbf{a}_{n}\right)_{k} e^{i \lambda k}}{\left(1-e^{i \lambda \mu}\right)^{n}\left(f(\lambda)+\lambda^{2 n} g(\lambda)\right)},
$$

where $\mathbf{S}_{\mu, p}$ is a linear operator in the space $\ell_{2}$ defined by the matrix with elements $\left(\mathbf{S}_{\mu, p}\right)_{l, k}=S_{l, p-\mu k}^{\mu}, l \geq 1,0 \leq k \leq n$, and $\left(\mathbf{S}_{\mu, N}\right)_{l, k}=0, l \geq 1, k>n$. The mean-square error of the estimate is calculated by the formula

$$
\begin{gathered}
\Delta(f, g ; \widehat{\xi}(-p))=\Delta(f, g ; \widehat{\eta}(-p))=\mathrm{E}|\eta(-p)-\widehat{\eta}(-p)|^{2} \\
=\frac{1}{2 \pi} \int_{-\pi}^{\pi} \frac{\left|e^{-i \lambda p}\left(1-e^{i \lambda \mu}\right)^{n} f(\lambda)+\lambda^{2 n} \sum_{k=1}^{\infty}\left(\mathbf{P}_{\mu}^{-1} \mathbf{S}_{\mu, p} \mathbf{a}_{n}\right)_{k} e^{i \lambda k}\right|^{2}}{\left|1-e^{i \lambda \mu}\right|^{2 n}\left(f(\lambda)+\lambda^{2 n} g(\lambda)\right)^{2}} g(\lambda) d \lambda
\end{gathered}
$$




$$
\begin{gathered}
+\frac{1}{2 \pi} \int_{-\pi}^{\pi} \frac{\left|e^{-i \lambda p}\left(1-e^{i \lambda \mu}\right)^{n} \lambda^{2 n} g(\lambda)-\lambda^{2 n} \sum_{k=1}^{\infty}\left(\mathbf{P}_{\mu}^{-1} \mathbf{S}_{\mu, p} \mathbf{a}_{n}\right)_{k} e^{i \lambda k}\right|^{2}}{\lambda^{2 n}\left|1-e^{i \lambda \mu}\right|^{2 n}\left(f(\lambda)+\lambda^{2 n} g(\lambda)\right)^{2}} f(\lambda) d \lambda \\
=\left\langle S_{\mu, p} \mathbf{a}_{n}, \mathbf{P}_{\mu}^{-1} \mathbf{S}_{\mu, p} \mathbf{a}_{n}\right\rangle+Q_{0,0} .
\end{gathered}
$$

Thus, we have the following statement.

\section{Corollary 3.1}

The optimal linear estimate $\widehat{\xi}(-p)$ of the unknown value $\xi(-p), p \geq 0$, of a stochastic sequence with $n$th stationary increments from observations of the sequence $\xi(k)+\eta(k)$ at points $k=0,-1,-2, \ldots$ is calculated by formula (20). The spectral characteristic $h_{\mu, p}(\lambda)$ of the optimal estimate $\widehat{\xi}(-p)$ is calculated by formula (21). The value of the mean-square error $\Delta(f, g ; \widehat{\xi}(-p))$ is calculated by formula (22).

Theorem 3.1, 3.1 and Corollary 3.1 determine solutions of the filtering problem for the linear functionals $A \xi, A_{N} \xi$ and the value $\xi(-p), p \geq 0$, using the Fourier coefficients of functions

$$
\frac{\lambda^{2 n}}{\left|1-e^{i \lambda \mu}\right|^{2 n}\left(f(\lambda)+\lambda^{2 n} g(\lambda)\right)}, \quad \frac{\lambda^{2 n} g(\lambda)}{\left|1-e^{i \lambda \mu}\right|^{2 n}\left(f(\lambda)+\lambda^{2 n} g(\lambda)\right)} .
$$

However, the problem of finding the inverse operator $\left(\mathbf{P}_{\mu}\right)^{-1}$ to the operator $\mathbf{P}_{\mu}$ defined by the Fourier coefficients of the function $\frac{\lambda^{2 n}}{\left|1-e^{i \lambda \mu}\right|^{2 n}\left(f(\lambda)+\lambda^{2 n} g(\lambda)\right)}$ is complicated in most cases. Therefore, we propose a method of finding the operator $\left(\mathbf{P}_{\mu}\right)^{-1}$ under the condition that the functions

$$
\frac{\left|1-e^{i \lambda \mu}\right|^{2 n}\left(f(\lambda)+\lambda^{2 n} g(\lambda)\right)}{\lambda^{2 n}}, \quad \frac{\lambda^{2 n}}{\left|1-e^{i \lambda \mu}\right|^{2 n}\left(f(\lambda)+\lambda^{2 n} g(\lambda)\right)}
$$

admit the canonical factorizations

$$
\begin{aligned}
\frac{\left|1-e^{i \lambda \mu}\right|^{2 n}\left(f(\lambda)+\lambda^{2 n} g(\lambda)\right)}{\lambda^{2 n}} & =\left|\sum_{k=0}^{\infty} \varphi_{\mu}(k) e^{-i \lambda k}\right|^{2}, \\
\frac{\lambda^{2 n}}{\left|1-e^{i \lambda \mu}\right|^{2 n}\left(f(\lambda)+\lambda^{2 n} g(\lambda)\right)} & =\left|\sum_{k=0}^{\infty} \psi_{\mu}(k) e^{-i \lambda k}\right|^{2} .
\end{aligned}
$$

Using coefficients $\varphi_{\mu}(k), \psi_{\mu}(k), k \geq 0$, from factorizations (24), (25), we define linear operators $\Phi_{\mu}$ and $\Psi_{\mu}$ in the space $\ell_{2}$. Let $\left(\Phi_{\mu}\right)_{k, j}=\varphi_{\mu}(k-j)$ and $\left(\Psi_{\mu}\right)_{k, j}=\psi_{\mu}(k-j)$ for $1 \leq j \leq k,\left(\Phi_{\mu}\right)_{k, j}=0$ and $\left(\Psi_{\mu}\right)_{k, j}=0$ for $j>k$, $k, j \geq 1$. The defined operators admit the following relation: $\Psi_{\mu} \Phi_{\mu}=\Phi_{\mu} \Psi_{\mu}=$ $I$, where $I$ is the identity operator. Moreover, the operator $\mathbf{P}_{\mu}$ admits the 
factorization $\mathbf{P}_{\mu}=\bar{\Psi}_{\mu}^{\prime} \Psi_{\mu}$. Thus, $\left(\mathbf{P}_{\mu}\right)^{-1}=\Phi_{\mu} \bar{\Phi}_{\mu}^{\prime}$ and elements of the matrix which determines the operator $\mathbf{V}_{\mu}=\left(\mathbf{P}_{\mu}\right)^{-1}$ are calculated by the formula

$$
V_{k, j}^{\mu}=\sum_{p=1}^{\min (k, j)} \varphi_{\mu}(k-p) \bar{\varphi}_{\mu}(j-p), \quad k, j \geq 1 .
$$

The following theorem holds true.

\section{Theorem 3.3}

Let functions (23) admit the canonical factorizations (24) and (25) respectively. In this case the inverse operator $\mathbf{P}_{\mu}^{-1}$ to the operator $\mathbf{P}_{\mu}$ is calculated by the formula $\mathbf{P}_{\mu}^{-1}=\Phi_{\mu} \bar{\Phi}_{\mu}^{\prime}$, where linear operator $\Phi_{\mu}$ in the space $\ell_{2}$ is determined by matrix with elements $\left(\Phi_{\mu}\right)_{k, j}=\varphi_{\mu}(k-j)$ if $1 \leq j \leq k$ and $\left(\Phi_{\mu}\right)_{k, j}=0$ if $j<k$, $k, j \geq 1$.

\section{Example 3.1}

Consider an $\operatorname{ARIMA}(0,1,1)$ sequence $\{\xi(m), m \in \mathbb{Z}\}$ whose first order increments are stationary and increments with step $\mu=1$ form an one-sided moving average sequence of order 1 with parameter $\phi$. The sequence $\xi(m)$ has the spectral density

$$
f(\lambda)=\frac{\lambda^{2}\left|1-\phi e^{-i \lambda}\right|^{2}}{\left|1-e^{-i \lambda}\right|^{2}} .
$$

Let $\{\eta(m), m \in \mathbb{Z}\}$ be an uncorrelated with $\xi(m)$ white noise stochastic sequence with mean 0 and variance 1 . The stochastic sequence $\{\xi(m)+\eta(m), m \in \mathbb{Z}\}$ is an $\operatorname{ARIMA}(0,1,1)$ sequence with the spectral density

$$
f(\lambda)+\lambda^{2} g(\lambda)=\frac{x \lambda^{2}\left|1-y e^{-i \lambda}\right|^{2}}{\left|1-e^{-i \lambda}\right|^{2}},
$$

where

$$
\begin{gathered}
x=\frac{1}{2}\left(3+\phi^{2} \mp \sqrt{\left(\phi^{2}-1\right)^{2}+(\phi-1)^{2}}\right), \\
y=\frac{1}{2 \phi+2}\left(3+\phi^{2} \pm \sqrt{\left(\phi^{2}-1\right)^{2}+(\phi-1)^{2}}\right) .
\end{gathered}
$$

Consider the problem of finding the mean square optimal linear estimate of the functional $A_{1} \xi=a \xi(0)+b \xi(-1)$ which depends on unknown values $\xi(0)$, $\xi(-1)$ of the sequence $\xi(m)$ from observations of the sequence $\xi(m)+\eta(m)$ at points $m=0,-1,-2, \ldots$. Condition (12) holds true if $|y|<1$. To calculate the spectral characteristic of the optimal estimate $\widehat{A}_{1} \xi$ of the functional $A_{1} \xi$ we use formula (17). The operator $\mathbf{P}_{\mu}=\mathbf{P}$ is determined by the matrix with elements $(\mathbf{P})_{l, k}=\frac{y^{p}}{x\left(1-y^{2}\right)}$, where $p=|k-l|, l, k \geq 1$. The inverse operator $\mathbf{V}=\mathbf{P}^{-1}$ is defined by the matrix with elements $(\mathbf{V})_{1,1}=x,(\mathbf{V})_{l, l}=x\left(1+y^{2}\right)$ 
if $l \geq 2,(\mathbf{V})_{l, k}=-x y$ if $|l-k|=1, l, k \geq 1$, and $(\mathbf{V})_{l, k}=0$ otherwise. The operator $\mathbf{S}_{\mu, N}=\mathbf{S}$ is defined by the matrix with elements $(\mathbf{S})_{l, m}=\frac{y^{l+m}}{x\left(1-y^{2}\right)}$ if $l \geq 1, m=-1,0,1$, and $(\mathbf{S})_{l, m}=0$ if $l \geq 1, m \geq 2$. The spectral characteristic $h_{1,1}(\lambda)$ of the estimate $\widehat{A}_{1} \xi$ is calculated by the formula

$$
h_{1,1}(\lambda)=\frac{1-e^{-i \lambda}}{i \lambda} \sum_{k=0}^{\infty} s(k) e^{-i \lambda k}
$$

where

$$
\begin{gathered}
s(0)=x^{-1}(a+b(y-1)), \\
s(k)=x^{-1} y^{k-1}\left(a y+b\left(y^{2}-y+1\right)\right), \quad k \geq 1 .
\end{gathered}
$$

The optimal estimate $\widehat{A}_{1} \xi$ of the functional $A_{1} \xi$ is calculated by the formula

$$
\begin{gathered}
\widehat{A}_{1} \xi=a(\xi(0)+\eta(0))+b(\xi(-1)+\eta(-1))-\sum_{k=0}^{\infty} s(k)\left(\xi^{(1)}(-k, 1)+\eta^{(1)}(-k, 1)\right) \\
=(a-s(0))(\xi(0)+\eta(0))+(b+s(0)-s(1))(\xi(-1)+\eta(-1)) \\
-\sum_{k=2}^{\infty}(s(k)-s(k-1))(\xi(-k)+\eta(-k)) \\
=x^{-1}(a(x-1)-b(y-1))(\xi(0)+\eta(0))+x^{-1}\left(a(1-y)-b\left(y^{2}-2 y-x+2\right)\right) \\
(\xi(-1)+\eta(-1))-\sum_{k=2}^{\infty} x^{-1} y^{k-2}(y-1)\left(a y+b\left(y^{2}-y+1\right)\right)(\xi(-k)+\eta(-k)) .
\end{gathered}
$$

In particular, the optimal estimate $\widehat{\xi}(0)$ of the value $\xi(0)$ is the following

$$
\widehat{\xi}(0)=\left(1-x^{-1}\right)(\xi(0)+\eta(0))-\sum_{k=1}^{\infty} x^{-1} y^{k-1}(y-1)(\xi(-k)+\eta(-k)) .
$$

\section{Filtering of cointegrated sequences}

Let $\{\xi(m), m \in \mathbb{Z}\}$ and $\{\zeta(m), m \in \mathbb{Z}\}$ be two integrated stochastic sequences of the same order $n$ which define stationary $n$th increment sequences $\xi^{(n)}(m, \mu)$ and $\zeta^{(n)}(m, \mu)$ with absolutely continuous spectral functions $F(\lambda)$ and $P(\lambda)$ which have spectral densities $f(\lambda)$ and $p(\lambda)$ correspondingly.

\section{Definition 4.1}

Two integrated stochastic sequences $\{\xi(m), m \in \mathbb{Z}\}$ and $\{\zeta(m), m \in \mathbb{Z}\}$ are called cointegrated if there exists a constant $\beta \neq 0$ such that the sequence $\{\zeta(m)-$ $\beta \xi(m): m \in \mathbb{Z}\}$ is stationary. 
Consider the filtering problem for cointegrated stochastic sequences which consists in finding the mean-square optimal linear estimates of the functionals

$$
A \xi=\sum_{k=0}^{\infty} a(k) \xi(-k), \quad A_{N} \xi=\sum_{k=0}^{N} a(k) \xi(-k)
$$

of unknown values of the sequence $\xi(m)$ from observations of the sequence $\zeta(m)$ at points $m=0,-1,-2, \ldots$. This problem can be solved by using results presented in the preceding section under the condition that sequences $\xi(m)$ and $\zeta(m)-\beta \xi(m)$ are uncorrelated.

Let the following condition holds true:

$$
\int_{-\pi}^{\pi} \frac{\lambda^{2 n}}{\left|1-e^{i \lambda \mu}\right|^{2 n} p(\lambda)} d \lambda<\infty .
$$

Determine operators $\mathbf{P}_{\mu}^{\beta}, \mathbf{S}_{\mu}^{\beta}, \mathbf{Q}^{\beta}$ with the help of the Fourier coefficients of functions

$$
\frac{\lambda^{2 n}}{\left|1-e^{i \lambda \mu}\right|^{2 n} p(\lambda)}, \quad \frac{\left(p(\lambda)-\beta^{2} f(\lambda)\right)}{\left|1-e^{i \lambda \mu}\right|^{2 n} p(\lambda)}, \quad \frac{f(\lambda) p(\lambda)-\beta^{2} f^{2}(\lambda)}{\lambda^{2 n} p(\lambda)}
$$

in the same way as operators $\mathbf{P}_{\mu}, \mathbf{S}_{\mu}, \mathbf{Q}$ are determined in Section 3. It follows from Theorem 3.1 that the spectral characteristic $h_{\mu}^{\beta}(\lambda)$ of the optimal estimate

$$
\widehat{A} \xi=A \zeta-\int_{-\pi}^{\pi} h_{\mu}^{\beta}(\lambda) d Z_{\zeta^{(n)}}(\lambda),
$$

of the functional $A \xi$ is calculated by the formula

$$
h_{\mu}^{\beta}(\lambda)=A\left(e^{-i \lambda}\right) \frac{p(\lambda)-\beta^{2} f(\lambda)}{(i \lambda)^{n} p(\lambda)}-\frac{(-i \lambda)^{n} \sum_{k=1}^{\infty}\left(\left(\mathbf{P}_{\mu}^{\beta}\right)^{-1} \mathbf{S}_{\mu}^{\beta} \mathbf{a}_{\mu}\right)_{k} e^{i \lambda k}}{\left(1-e^{i \lambda \mu}\right)^{n} p(\lambda)} .
$$

The mean-square error of the estimate is calculated by the formula

$$
\begin{gathered}
\Delta(f, g ; \widehat{A} \xi) \\
=\frac{1}{2 \pi} \int_{-\pi}^{\pi} \frac{\left|A\left(e^{-i \lambda}\right)\left(1-e^{i \lambda \mu}\right)^{n} \beta^{2} f(\lambda)+\lambda^{2 n} \sum_{k=1}^{\infty}\left(\left(\mathbf{P}_{\mu}^{\beta}\right)^{-1} \mathbf{S}_{\mu}^{\beta} \mathbf{a}_{\mu}\right)_{k} e^{i \lambda k}\right|^{2}}{\lambda^{2 n}\left|1-e^{i \lambda \mu}\right|^{2 n} p^{2}(\lambda)} p(\lambda) d \lambda \\
-\frac{\beta^{2}}{2 \pi} \int_{-\pi}^{\pi} \frac{\left|A\left(e^{-i \lambda}\right)\left(1-e^{i \lambda \mu}\right)^{n} \beta^{2} f(\lambda)+\lambda^{2 n} \sum_{k=1}^{\infty}\left(\left(\mathbf{P}_{\mu}^{\beta}\right)^{-1} \mathbf{S}_{\mu}^{\beta} \mathbf{a}_{\mu}\right)_{k} e^{i \lambda k}\right|^{2}}{\lambda^{2 n}\left|1-e^{i \lambda \mu}\right|^{2 n} p^{2}(\lambda)} f(\lambda) d \lambda \\
+\frac{1}{2 \pi} \int_{-\pi}^{\pi} \frac{\left|A\left(e^{-i \lambda}\right)\left(1-e^{i \lambda \mu}\right)^{n}\left(p(\lambda)-\beta^{2} f(\lambda)\right)-\lambda^{2 n} \sum_{k=1}^{\infty}\left(\left(\mathbf{P}_{\mu}^{\beta}\right)^{-1} \mathbf{S}_{\mu}^{\beta} \mathbf{a}_{\mu}\right)_{k} e^{i \lambda k}\right|^{2}}{\lambda^{2 n}\left|1-e^{i \lambda \mu}\right|^{2 n} p^{2}(\lambda)} \\
f(\lambda) d \lambda=\left\langle\mathbf{S}_{\mu}^{\beta} \mathbf{a}_{\mu},\left(\mathbf{P}_{\mu}^{\beta}\right)^{-1} \mathbf{S}_{\mu}^{\beta} \mathbf{a}_{\mu}\right\rangle+\left\langle\mathbf{Q}^{\beta} \mathbf{a}, \mathbf{a}\right\rangle,
\end{gathered}
$$

In summary we have the following statement. 


\section{Theorem 4.1}

Let the cointegrated stochastic sequences $\{\xi(m), m \in \mathbb{Z}\}$ and $\{\zeta(m), m \in \mathbb{Z}\}$ have absolutely continuous spectral functions $F(\lambda)$ and $G(\lambda)$ which have spectral densities $f(\lambda)$ and $p(\lambda)$ satisfying conditions (26). Let coefficients $\left\{a_{\mu}(k): k \geq\right.$ $-\mu n\}$ determined by formula (13) satisfy conditions (8). If the sequences $\xi(m)$ and $\zeta(m)-\beta \xi(m)$ are uncorrelated, then the optimal linear estimate $\widehat{A} \xi$ of the functional $A \xi$ of unknown elements $\xi(m), m \leq 0$, from observations of the sequence $\zeta(m)$ at points $m=0,-1,-2, \ldots$ is calculated by formula (28). The spectral characteristic $h_{\mu}^{\beta}(\lambda)$ of the optimal estimate $\widehat{A} \xi$ is calculated by formula (29). The value of the mean-square error $\Delta(f, g ; \widehat{A} \xi)$ is calculated by formula (30).

Let operators $\mathbf{P}_{\mu}^{\beta}, \mathbf{S}_{\mu, N}^{\beta}, \mathbf{Q}_{N}^{\beta}$ be determined by the Fourier coefficients of functions (27) in the same way as operators $\mathbf{P}_{\mu}, \mathbf{S}_{\mu, N}, \mathbf{Q}_{N}$ are determined in Section 3. It follows from Theorem 3.2 that the spectral characteristic $h_{\mu, N}^{\beta}(\lambda)$ of the optimal estimate

$$
\widehat{A}_{N} \xi=A_{N} \zeta-\int_{-\pi}^{\pi} h_{\mu, N}^{\beta}(\lambda) d Z_{\zeta^{(n)}}(\lambda)
$$

of the functional $A_{N} \xi$ is calculated by the formula

$$
h_{\mu, N}^{\beta}(\lambda)=A_{N}\left(e^{-i \lambda}\right) \frac{p(\lambda)-\beta^{2} f(\lambda)}{(i \lambda)^{n} p(\lambda)}-\frac{(-i \lambda)^{n} \sum_{k=1}^{\infty}\left(\left(\mathbf{P}_{\mu}^{\beta}\right)^{-1} \mathbf{S}_{\mu, N}^{\beta} \mathbf{a}_{\mu, N}\right)_{k} e^{i \lambda k}}{\left(1-e^{i \lambda \mu}\right)^{n} p(\lambda)} .
$$

The mean-square error of the estimate $\widehat{A}_{N} \xi$ is calculated by the formula

$$
\begin{gathered}
\Delta\left(f, g ; \widehat{A}_{N} \xi\right) \\
=\frac{1}{2 \pi} \int_{-\pi}^{\pi} \frac{\left|A_{N}\left(e^{-i \lambda}\right)\left(1-e^{i \lambda \mu}\right)^{n} \beta^{2} f(\lambda)+\lambda^{2 n} \sum_{k=1}^{\infty}\left(\left(\mathbf{P}_{\mu}^{\beta}\right)^{-1} \mathbf{S}_{\mu, N}^{\beta} \mathbf{a}_{\mu, N}\right)_{k} e^{i \lambda k}\right|^{2}}{\lambda^{2 n}\left|1-e^{i \lambda \mu}\right|^{2 n} p^{2}(\lambda)} p(\lambda) d \lambda \\
-\frac{\beta^{2}}{2 \pi} \int_{-\pi}^{\pi} \frac{\left|A_{N}\left(e^{-i \lambda}\right)\left(1-e^{i \lambda \mu}\right)^{n} \beta^{2} f(\lambda)+\lambda^{2 n} \sum_{k=1}^{\infty}\left(\left(\mathbf{P}_{\mu}^{\beta}\right)^{-1} \mathbf{S}_{\mu, N}^{\beta} \mathbf{a}_{\mu, N}\right)_{k} e^{i \lambda k}\right|^{2}}{\lambda^{2 n}\left|1-e^{i \lambda \mu}\right|^{2 n} p^{2}(\lambda)} f(\lambda) d \lambda \\
+\frac{1}{2 \pi} \int_{-\pi}^{\pi} \frac{\left|A_{N}\left(e^{-i \lambda}\right)\left(1-e^{i \lambda \mu}\right)^{n}\left(p(\lambda)-\beta^{2} f(\lambda)\right)-\lambda^{2 n} \sum_{k=1}^{\infty}\left(\left(\mathbf{P}_{\mu}^{\beta}\right)^{-1} \mathbf{S}_{\mu, N}^{\beta} \mathbf{a}_{\mu, N}\right)_{k} e^{i \lambda k}\right|^{2}}{\lambda^{2 n}\left|1-e^{i \lambda \mu}\right|^{2 n} p^{2}(\lambda)} \\
f(\lambda) d \lambda=\left\langle\mathbf{S}_{\mu, N}^{\beta} \mathbf{a}_{\mu, N},\left(\mathbf{P}_{\mu}^{\beta}\right)^{-1} \mathbf{S}_{\mu, N}^{\beta} \mathbf{a}_{\mu, N}\right\rangle+\left\langle\mathbf{Q}_{N}^{\beta} \mathbf{a}_{N}, \mathbf{a}_{N}\right\rangle .
\end{gathered}
$$

The following theorem holds true.

\section{Theorem 4.2}

Let the cointegrated stochastic sequences $\{\xi(m), m \in \mathbb{Z}\}$ and $\{\zeta(m), m \in \mathbb{Z}\}$ have absolutely continuous spectral functions $F(\lambda)$ and $G(\lambda)$ which have spectral 
densities $f(\lambda)$ and $p(\lambda)$ satisfying condition (26). If the sequences $\xi(m)$ and $\zeta(m)-\beta \xi(m)$ are uncorrelated, then the optimal linear estimate $\widehat{A}_{N} \xi$ of the functional $A_{N} \xi$ of unknown elements $\xi(m),-N \leq m \leq 0$, from observations of the sequence $\zeta(m)$ at points $m=0,-1,-2, \ldots$ is calculated by formula (31). The spectral characteristic $h_{\mu, N}^{\beta}(\lambda)$ of the optimal estimate $\widehat{A}_{N} \xi$ is calculated by formula (32). The value of the mean-square error $\Delta\left(f, g ; \widehat{A}_{N} \xi\right)$ is calculated by formula (33).

\section{Minimax-robust method of filtering}

The values of mean-square errors $\Delta\left(h_{\mu}(f, g) ; f, g\right):=\Delta(f, g ; \widehat{A} \xi)$ and $\Delta\left(h_{\mu, N}(f, g) ; f, g\right):=\Delta\left(f, g ; \widehat{A}_{N} \xi\right)$ and spectral characteristics $h_{\mu}(f, g)$ and $h_{\mu, N}(f, g)$ of the optimal linear estimates $\widehat{A} \xi$ and $\widehat{A}_{N} \xi$ of the functionals $A \xi$ and $A_{N} \xi$ of unknown values of the sequence $\xi(m)$ based on observations of the stochastic sequence $\xi(k)+\eta(k)$ are derived by formulas (15), (14) and (19), (17) correspondingly under the condition that spectral densities $f(\lambda)$ and $g(\lambda)$ of stochastic sequences $\xi(m)$ and $\eta(m)$ are known. In the case where spectral densities are not exactly known, but a set $\mathcal{D}=\mathcal{D}_{f} \times \mathcal{D}_{g}$ of admissible spectral densities is given, the minimax (robust) approach to estimation of functionals which depend on the unknown values of stochastic sequence with stationary increments is reasonable. In other words we are interested in finding an estimate that minimizes the maximum of mean-square errors for all spectral densities from a given class $\mathcal{D}=\mathcal{D}_{f} \times \mathcal{D}_{g}$ of admissible spectral densities simultaneously.

\section{Definition 5.1}

For a given class of spectral densities $\mathcal{D}=\mathcal{D}_{f} \times \mathcal{D}_{g}$ spectral densities $f_{0}(\lambda) \in \mathcal{D}_{f}$, $g_{0}(\lambda) \in \mathcal{D}_{g}$ are called least favorable in the class $\mathcal{D}$ for the optimal linear filtering of the functional $A \xi$ if the following relation holds true

$$
\Delta\left(f_{0}, g_{0}\right)=\Delta\left(h\left(f_{0}, g_{0}\right) ; f_{0}, g_{0}\right)=\max _{(f, g) \in \mathcal{D}_{f} \times \mathcal{D}_{g}} \Delta(h(f, g) ; f, g) .
$$

\section{Definition 5.2}

For a given class of spectral densities $\mathcal{D}=\mathcal{D}_{f} \times \mathcal{D}_{g}$ the spectral characteristic $h^{0}(\lambda)$ of the optimal linear estimate of the functional $A \xi$ is called minimax-robust if there are satisfied conditions

$$
\begin{gathered}
h^{0}(\lambda) \in H_{\mathcal{D}}=\bigcap_{(f, g) \in \mathcal{D}_{f} \times \mathcal{D}_{g}} L_{2}^{0}\left(f(\lambda)+\lambda^{2 n} g(\lambda)\right), \\
\min _{h \in H_{\mathcal{D}}} \max _{(f, g) \in \mathcal{D}_{f} \times \mathcal{D}_{g}} \Delta(h ; f, g)=\max _{(f, g) \in \mathcal{D}_{f} \times \mathcal{D}_{g}} \Delta\left(h^{0} ; f, g\right) .
\end{gathered}
$$


Using the derived in the previous sections formulas and the introduced definitions we can conclude that the following statement holds true.

\section{Lemma 5.1}

Spectral densities $f^{0} \in \mathcal{D}_{f}, g^{0} \in \mathcal{D}_{g}$ which satisfy condition (12) are least favorable in the class $\mathcal{D}=\mathcal{D}_{f} \times \mathcal{D}_{g}$ for the optimal linear filtering of the functional $A \xi$ if operators $\mathbf{P}_{\mu}^{0}, \mathbf{S}_{\mu}^{0}, \mathbf{Q}^{0}$ determined by the Fourier coefficients of the functions

$$
\frac{\lambda^{2 n}}{\left|1-e^{i \lambda \mu}\right|^{2 n}\left(f^{0}(\lambda)+\lambda^{2 n} g^{0}(\lambda)\right)}, \frac{\lambda^{2 n} g^{0}(\lambda)}{\left|1-e^{i \lambda \mu}\right|^{2 n}\left(f^{0}(\lambda)+\lambda^{2 n} g^{0}(\lambda)\right)}, \frac{f^{0}(\lambda) g^{0}(\lambda)}{f^{0}(\lambda)+\lambda^{2 n} g^{0}(\lambda)}
$$

determine a solution of the conditional extremum problem

$$
\max _{f \in \mathcal{D}}\left(\left\langle\mathbf{S}_{\mu} \mathbf{a}_{\mu}, \mathbf{P}_{\mu}^{-1} \mathbf{S}_{\mu} \mathbf{a}_{\mu}\right\rangle+\langle\mathbf{Q} \mathbf{a}, \mathbf{a}\rangle\right)=\left\langle\mathbf{S}_{\mu}^{0} \mathbf{a}_{\mu},\left(\mathbf{P}_{\mu}^{0}\right)^{-1} \mathbf{S}_{\mu}^{0} \mathbf{a}_{\mu}\right\rangle+\left\langle\mathbf{Q}^{0} \mathbf{a}, \mathbf{a}\right\rangle .
$$

The minimax spectral characteristic is determined as $h^{0}=h_{\mu}\left(f^{0}, g^{0}\right)$ if $h_{\mu}\left(f^{0}, g^{0}\right) \in H_{\mathcal{D}}$.

The function $h^{0}$ and the pair $\left(f^{0}, g^{0}\right)$ form a saddle point of the function $\Delta(h ; f, g)$ on the set $H_{\mathcal{D}} \times \mathcal{D}$. The saddle point inequalities

$$
\Delta\left(h ; f^{0}, g^{0}\right) \geq \Delta\left(h^{0} ; f^{0}, g^{0}\right) \geq \Delta\left(h^{0} ; f, g\right) \quad \forall f \in \mathcal{D}_{f}, \forall g \in \mathcal{D}_{g}, \forall h \in H_{\mathcal{D}}
$$

hold true if $h^{0}=h_{\mu}\left(f^{0}, g^{0}\right)$ and $h_{\mu}\left(f^{0}, g^{0}\right) \in H_{\mathcal{D}}$, where $\left(f^{0}, g^{0}\right)$ is a solution of the conditional extremum problem:

$$
\begin{gathered}
\widetilde{\Delta}(f, g)=-\Delta\left(h_{\mu}\left(f^{0}, g^{0}\right) ; f, g\right) \rightarrow \inf , \quad(f, g) \in \mathcal{D}, \\
\Delta\left(h_{\mu}\left(f^{0}, g^{0}\right) ; f, g\right) \\
=\frac{1}{2 \pi} \int_{-\pi}^{\pi} \frac{\left|A\left(e^{-i \lambda}\right)\left(1-e^{i \lambda \mu}\right)^{n} f^{0}(\lambda)+\lambda^{2 n} \sum_{k=1}^{\infty}\left(\left(\mathbf{P}_{\mu}^{0}\right)^{-1} \mathbf{S}_{\mu}^{0} \mathbf{a}_{\mu}\right)_{k} e^{i \lambda k}\right|^{2}}{\left|1-e^{i \lambda \mu}\right|^{2 n}\left(f^{0}(\lambda)+\lambda^{2 n} g^{0}(\lambda)\right)^{2}} g(\lambda) d \lambda \\
+\frac{1}{2 \pi} \int_{-\pi}^{\pi} \frac{\left|A\left(e^{-i \lambda}\right)\left(1-e^{i \lambda \mu}\right)^{n} \lambda^{2 n} g^{0}(\lambda)-\lambda^{2 n} \sum_{k=1}^{\infty}\left(\left(\mathbf{P}_{\mu}^{0}\right)^{-1} \mathbf{S}_{\mu}^{0} \mathbf{a}_{\mu}\right)_{k} e^{i \lambda k}\right|^{2}}{\lambda^{2 n}\left|1-e^{i \lambda \mu}\right|^{2 n}\left(f^{0}(\lambda)+\lambda^{2 n} g^{0}(\lambda)\right)^{2}} f(\lambda) d \lambda .
\end{gathered}
$$

This conditional extremum problem is equivalent to the unconditional extremum problem

$$
\Delta_{\mathcal{D}}(f, g)=\widetilde{\Delta}(f, g)+\delta\left(f, g \mid \mathcal{D}_{f} \times \mathcal{D}_{g}\right) \rightarrow \inf ,
$$

where $\delta\left(f, g \mid \mathcal{D}_{f} \times \mathcal{D}_{g}\right)$ is the indicator function of the set $\mathcal{D}_{f} \times \mathcal{D}_{g}$. Solution $\left(f^{0}, g^{0}\right)$ to this unconditional extremum problem is characterized by condition $0 \in \partial \Delta_{\mathcal{D}}\left(f^{0}, g^{0}\right)$ (see [24, 34]). 


\section{Least favorable spectral densities in the class $\mathcal{D}_{f}^{0} \times \mathcal{D}_{g}^{0}$}

Consider the problem of minimax filtering of the functional $A \xi$ for the set of admissible spectral densities $\mathcal{D}=\mathcal{D}_{f}^{0} \times \mathcal{D}_{g}^{0}$, where

$$
\mathcal{D}_{f}^{0}=\left\{f(\lambda) \mid \frac{1}{2 \pi} \int_{-\pi}^{\pi} f(\lambda) d \lambda \leq P_{1}\right\}, \quad \mathcal{D}_{g}^{0}=\left\{g(\lambda) \mid \frac{1}{2 \pi} \int_{-\pi}^{\pi} g(\lambda) d \lambda \leq P_{2}\right\} .
$$

Let us assume that densities $f^{0} \in \mathcal{D}_{f}^{0}, g^{0} \in \mathcal{D}_{g}^{0}$ and functions

$$
\begin{aligned}
h_{\mu, f}\left(f^{0}, g^{0}\right) & =\frac{\left|A\left(e^{-i \lambda}\right)\left(1-e^{i \lambda \mu}\right)^{n} \lambda^{2 n} g^{0}(\lambda)-\lambda^{2 n} \sum_{k=1}^{\infty}\left(\left(\mathbf{P}_{\mu}^{0}\right)^{-1} \mathbf{S}_{\mu}^{0} \mathbf{a}_{\mu}\right)_{k} e^{i \lambda k}\right|}{|\lambda|^{n}\left|1-e^{i \lambda \mu}\right|^{n}\left(f^{0}(\lambda)+\lambda^{2 n} g^{0}(\lambda)\right)}, \\
h_{\mu, g}\left(f^{0}, g^{0}\right) & =\frac{\left|A\left(e^{-i \lambda}\right)\left(1-e^{i \lambda \mu}\right)^{n} f^{0}(\lambda)+\lambda^{2 n} \sum_{k=1}^{\infty}\left(\left(\mathbf{P}_{\mu}^{0}\right)^{-1} \mathbf{S}_{\mu}^{0} \mathbf{a}_{\mu}\right)_{k} e^{i \lambda k}\right|}{\left|1-e^{i \lambda \mu}\right|^{n}\left(f^{0}(\lambda)+\lambda^{2 n} g^{0}(\lambda)\right)}
\end{aligned}
$$

are bounded. In this case the functional $\Delta\left(h_{\mu}\left(f^{0}, g^{0}\right) ; f, g\right)$ is continuous and bounded in the $\mathcal{L}_{1} \times \mathcal{L}_{1}$ space. It comes from the condition $0 \in \partial \Delta_{\mathcal{D}}\left(f^{0}, g^{0}\right)$ that least favorable densities $f^{0} \in \mathcal{D}_{f}^{0}, g^{0} \in \mathcal{D}_{g}^{0}$ satisfy the equation

$$
\begin{gathered}
\left|A\left(e^{-i \lambda}\right)\left(1-e^{i \lambda \mu}\right)^{n} \lambda^{2 n} g^{0}(\lambda)-\lambda^{2 n} \sum_{k=1}^{\infty}\left(\left(\mathbf{P}_{\mu}^{0}\right)^{-1} \mathbf{S}_{\mu}^{0} \mathbf{a}_{\mu}\right)_{k} e^{i \lambda k}\right| \\
=\alpha_{1}|\lambda|^{n}\left|1-e^{i \lambda \mu}\right|^{n}\left(f^{0}(\lambda)+\lambda^{2 n} g^{0}(\lambda)\right), \\
\left|A\left(e^{-i \lambda}\right)\left(1-e^{i \lambda \mu}\right)^{n} f^{0}(\lambda)+\lambda^{2 n} \sum_{k=1}^{\infty}\left(\left(\mathbf{P}_{\mu}^{0}\right)^{-1} \mathbf{S}_{\mu}^{0} \mathbf{a}_{\mu}\right)_{k} e^{i \lambda k}\right| \\
=\alpha_{2}\left|1-e^{i \lambda \mu}\right|^{n}\left(f^{0}(\lambda)+\lambda^{2 n} g^{0}(\lambda)\right),
\end{gathered}
$$

where $\alpha_{1} \geq 0$ and $\alpha_{2} \geq 0$ are constants such that $\alpha_{1} \neq 0$ if $\frac{1}{2 \pi} \int_{-\pi}^{\pi} f^{0}(\lambda) d \lambda=P_{1}$ and $\alpha_{2} \neq 0$ if $\frac{1}{2 \pi} \int_{-\pi}^{\pi} g^{0}(\lambda) d \lambda=P_{2}$.

Thus, we have the following statements.

\section{Theorem 6.1}

Let spectral densities $f^{0}(\lambda) \in \mathcal{D}_{f}^{0}$ and $g^{0}(\lambda) \in \mathcal{D}_{g}^{0}$ satisfy condition (12), let functions $h_{\mu, f}\left(f^{0}, g^{0}\right)$ and $h_{\mu, g}\left(f^{0}, g^{0}\right)$ be bounded. The spectral densities $f^{0}(\lambda)$ and $g^{0}(\lambda)$ determined by equations (37), (38) are least favorable in the class $\mathcal{D}=$ $\mathcal{D}_{f}^{0} \times \mathcal{D}_{g}^{0}$ for the optimal linear estimation of the functional $A \xi$ if they determine solution of extremum problem (34). The function $h_{\mu}\left(f^{0}, g^{0}\right)$ determined by formula (14) is minimax spectral characteristic of the optimal estimate of the functional $A \xi$. 


\section{Theorem 6.2}

Let spectral density $f(\lambda)$ be known, let spectral density $g^{0}(\lambda) \in \mathcal{D}_{g}^{0}$ and let conditions (12) be satisfied. Let the function $h_{\mu, g}\left(f, g^{0}\right)$ be bounded. Spectral density $g^{0}(\lambda)$ is least favorable in the class $\mathcal{D}_{g}^{0}$ for the optimal linear filtering of the functional $A \xi$ if it is of the form

$g^{0}(\lambda)=\frac{1}{\lambda^{2 n}} \max \left\{0, \frac{\left|A\left(e^{-i \lambda}\right)\left(1-e^{i \lambda \mu}\right)^{n} f(\lambda)+\lambda^{2 n} \sum_{k=1}^{\infty}\left(\left(\mathbf{P}_{\mu}^{0}\right)^{-1} \mathbf{S}_{\mu}^{0} \mathbf{a}_{\mu}\right)_{k} e^{i \lambda k}\right|}{\alpha_{2}\left|1-e^{i \lambda \mu}\right|^{n}}-f(\lambda)\right\}$

and the pair $\left(f, g^{0}\right)$ determines a solution of the extremum problem (34). The function $h_{\mu}\left(f, g^{0}\right)$ determined by formula (14) is minimax spectral characteristic of the optimal estimation of the functional $A \xi$.

\section{Theorem 6.3}

Let spectral density $g(\lambda)$ be known, let spectral density $f^{0}(\lambda) \in \mathcal{D}_{f}^{0}$ and let condition (12) be satisfied. Let the function $h_{\mu, f}\left(f^{0}, g\right)$ be bounded. Spectral density $f^{0}(\lambda)$ is least favorable in the class $\mathcal{D}_{f}^{0}$ for the optimal linear filtering of the functional $A \xi$ if it is of the form

$$
f^{0}(\lambda)=\max \left\{0, \frac{|\lambda|^{n}\left|A\left(e^{-i \lambda}\right)\left(1-e^{i \lambda \mu}\right)^{n} g(\lambda)-\sum_{k=1}^{\infty}\left(\left(\mathbf{P}_{\mu}^{0}\right)^{-1} \mathbf{S}_{\mu}^{0} \mathbf{a}_{\mu}\right)_{k} e^{i \lambda k}\right|}{\alpha_{1}\left|1-e^{i \lambda \mu}\right|^{n}}-\lambda^{2 n} g(\lambda)\right\}
$$

and the pair $\left(f^{0}, g\right)$ determines a solution of the extremum problem (34). The function $h_{\mu}\left(f^{0}, g\right)$ determined by formula (14) is minimax spectral characteristic of the optimal estimation of the functional $A \xi$.

\section{Least favorable densities in the class $\mathcal{D}=\mathcal{D}_{u}^{v} \times \mathcal{D}_{\varepsilon}$}

Consider the problem of optimal linear filtering of the functional $A \xi$ for the set of spectral densities $\mathcal{D}=\mathcal{D}_{u}^{v} \times \mathcal{D}_{\varepsilon}$, where

$$
\begin{gathered}
\mathcal{D}_{u}^{v}=\left\{f(\lambda) \mid v(\lambda) \leq f(\lambda) \leq u(\lambda), \frac{1}{2 \pi} \int_{-\pi}^{\pi} f(\lambda) d \lambda \leq P_{1}\right\}, \\
\mathcal{D}_{\varepsilon}=\left\{g(\lambda) \mid g(\lambda)=(1-\varepsilon) g_{1}(\lambda)+\varepsilon w(\lambda), \frac{1}{2 \pi} \int_{-\pi}^{\pi} g(\lambda) d \lambda \leq P_{2}\right\} .
\end{gathered}
$$

Here spectral densities $u(\lambda), v(\lambda), g_{1}(\lambda)$ are known and fixed, and spectral densities $u(\lambda), v(\lambda)$ are bounded.

Let spectral densities $f^{0} \in \mathcal{D}_{u}^{v}, g^{0} \in \mathcal{D}_{\varepsilon}$ be such that functions $h_{\mu, f}\left(f^{0}, g^{0}\right)$ and $h_{\mu, g}\left(f^{0}, g^{0}\right)$ determined by formulas (35), (36) are bounded. From the condition $0 \in \partial \Delta_{\mathcal{D}}\left(f^{0}, g^{0}\right)$ we find the following equations that define least favorable densities

$$
\left|A\left(e^{-i \lambda}\right)\left(1-e^{i \lambda \mu}\right)^{n} \lambda^{2 n} g^{0}(\lambda)-\lambda^{2 n} \sum_{k=1}^{\infty}\left(\left(\mathbf{P}_{\mu}^{0}\right)^{-1} \mathbf{S}_{\mu}^{0} \mathbf{a}_{\mu}\right)_{k} e^{i \lambda k}\right|
$$




$$
\begin{gathered}
=\alpha_{1}|\lambda|^{n}\left|1-e^{i \lambda \mu}\right|^{n}\left(f^{0}(\lambda)+\lambda^{2 n} g^{0}(\lambda)\right)\left(\gamma_{1}(\lambda)+\gamma_{2}(\lambda)+\alpha_{1}^{-1}\right), \\
\left|A\left(e^{-i \lambda}\right)\left(1-e^{i \lambda \mu}\right)^{n} f^{0}(\lambda)+\lambda^{2 n} \sum_{k=1}^{\infty}\left(\left(\mathbf{P}_{\mu}^{0}\right)^{-1} \mathbf{S}_{\mu}^{0} \mathbf{a}_{\mu}\right)_{k} e^{i \lambda k}\right| \\
=\alpha_{2}\left|1-e^{i \lambda \mu}\right|^{n}\left(f^{0}(\lambda)+\lambda^{2 n} g^{0}(\lambda)\right)\left(\varphi(\lambda)+\alpha_{2}^{-1}\right),
\end{gathered}
$$

where $\gamma_{1} \leq 0$ and $\gamma_{1}=0$ if $f^{0}(\lambda) \geq v(\lambda) ; \gamma_{2}(\lambda) \geq 0$ and $\gamma_{2}=0$ if $f^{0}(\lambda) \leq u(\lambda)$; $\varphi(\lambda) \leq 0$ and $\varphi(\lambda)=0$ when $g^{0}(\lambda) \geq(1-\varepsilon) g_{1}(\lambda)$.

The following statements hold true.

\section{Theorem 7.1}

Let spectral densities $f^{0}(\lambda) \in \mathcal{D}_{u}^{v}, \quad g^{0}(\lambda) \in \mathcal{D}_{\varepsilon}$ satisfy condition (12). Let functions $h_{\mu, f}\left(f^{0}, g^{0}\right)$ and $h_{\mu, g}\left(f^{0}, g^{0}\right)$ determined by formulas (35), (36) be bounded. Spectral densities $f^{0}(\lambda)$ and $g^{0}(\lambda)$ determined by equations (39), (40) are least favorable in the class $\mathcal{D}=\mathcal{D}_{u}^{v} \times \mathcal{D}_{\varepsilon}$ for the optimal linear filtering of the functional $A \xi$ if they determine a solution of extremum problem (34). The function $h_{\mu}\left(f^{0}, g^{0}\right)$ determined by (14) is minimax spectral characteristic of the optimal estimate of the functional $A \xi$.

\section{Theorem 7.2}

Let spectral density $f(\lambda)$ be known, let spectral density $g^{0}(\lambda) \in \mathcal{D}_{\varepsilon}$ and let condition (12) be satisfied. Assume that the function $h_{\mu, g}\left(f, g^{0}\right)$ determined by formula (36) is bounded. Spectral density $g^{0}(\lambda)$ is least favorable in the class $\mathcal{D}_{\varepsilon}$ for the optimal linear filtering of the functional $A \xi$ if it is of the form

$$
\begin{gathered}
g^{0}(\lambda)=\frac{1}{\lambda^{2 n}} \max \left\{(1-\varepsilon) g_{1}(\lambda), f_{1}(\lambda)\right\} \\
f_{1}(\lambda)=\frac{\left|A\left(e^{-i \lambda}\right)\left(1-e^{i \lambda \mu}\right)^{n} f(\lambda)+\lambda^{2 n} \sum_{k=1}^{\infty}\left(\left(\mathbf{P}_{\mu}^{0}\right)^{-1} \mathbf{S}_{\mu}^{0} \mathbf{a}_{\mu}\right)_{k} e^{i \lambda k}\right|}{\alpha_{2}\left|1-e^{i \lambda \mu}\right|^{n}}-f(\lambda),
\end{gathered}
$$

and the pair $\left(f, g^{0}\right)$ determines a solution of extremum problem (34). The function $h_{\mu}\left(f, g^{0}\right)$ determined by formula (14) is minimax spectral characteristic of the optimal estimate of the functional $A \xi$.

\section{Theorem 7.3}

Let spectral density $g(\lambda)$ be known, let spectral density $f^{0}(\lambda) \in \mathcal{D}_{u}^{v}$ and let condition (12) be satisfied. Let the function $h_{\mu, f}\left(f^{0}, g\right)$ be bounded. Spectral density $f^{0}(\lambda)$ is least favorable in the class $\mathcal{D}_{u}^{v}$ for the optimal linear filtering of the functional $A \xi$ if it is of the form

$$
\begin{gathered}
f^{0}(\lambda)=\min \left\{v(\lambda), \max \left\{u(\lambda), g_{2}(\lambda)\right\}\right\}, \\
g_{2}(\lambda)=\frac{|\lambda|^{n}\left|A\left(e^{-i \lambda}\right)\left(1-e^{i \lambda \mu}\right)^{n} g(\lambda)-\sum_{k=1}^{\infty}\left(\left(\mathbf{P}_{\mu}^{0}\right)^{-1} \mathbf{S}_{\mu}^{0} \mathbf{a}_{\mu}\right)_{k} e^{i \lambda k}\right|}{\alpha_{1}\left|1-e^{i \lambda \mu}\right|^{n}}-\lambda^{2 n} g(\lambda)
\end{gathered}
$$


and the pair $\left(f^{0}, g\right)$ determines a solution of extremum problem (34). The function $h_{\mu}\left(f^{0}, g\right)$ determined by formula (14) is minimax spectral characteristic of the optimal estimation of the functional $A \xi$.

\section{Conclusions}

In this article we propose solutions of the filtering problem for functionals $A \xi$ and $A_{N} \xi$ which depend on unobserved values of a stochastic sequence $\xi(k)$ with stationary $n$th increments. Estimates are based on observations of the sequence $\xi(k)+\eta(k)$ at points of time $k=0,-1,-2, \ldots$, where $\eta(k)$ is an uncorrelated with $\xi(k)$ stationary sequence. We derived formulas for calculating values of the mean-square errors and spectral characteristics of the optimal linear estimates of the functionals in the case where spectral densities of the sequences are known. The obtained results are applied to find solution of the filtering problem for cointegrated sequences. In the case of spectral uncertainty where spectral densities are not known exactly, but a set of admissible spectral densities is specified, the minimax-robust method is applied. Formulas that determine least favorable spectral densities and minimax (robust) spectral characteristics are derived for some special sets of admissible spectral densities. The filtering problem for $\operatorname{ARIMA}(0,1,1)$ sequence is analyzed as an example of application of the developed method.

\section{REFERENCES}

1. G. E. P. Box, G. M. Jenkins, G. C. Reinsel, Time series analysis. Forecasting and control. 3rd ed. Englewood Cliffs, NJ: Prentice Hall, 1994.

2. I. I. GiKhman, A. V. SKOROKHOD, The theory of stochastic processes. I. Berlin: Springer, 2004.

3. I.I. Dubovets'Ka, O.Yu. MasyutKa, M.P. Moklyachuk, Interpolation of periodically correlated stochastic sequences, Theor. Probability and Math. Statist., 84(2012), 43-56.

4. I. I. Dubovets'Ka, M.P. Moklyachuk, Filtration of linear functionals of periodically correlated sequences, Theor. Probability and Math. Statist., 86(2013), 51-64.

5. I. I. Dubovets'KA, M. P. MOKLYACHUK, Filtering of periodically correlated processes, Prykl. Stat., Aktuarna Finans. Mat., (2012), No. 2, 149-158.

6. I. I. Dubovets'KA, M. P. Moklyachuk, Extrapolation of periodically correlated processes from observations with noise, Theor. Probability and Math. Statist., 88(2013), 4355.

7. I. I. Dubovets'Ka, M. P. Moklyachuk, Minimax estimation problem for periodically correlated stochastic processes, Journal of Mathematics and System Science, 3(2013), No. 1, 26-30.

8. I. I. Dubovets'KA, M. P. Moklyachuk, On minimax estimation problems for periodically correlated stochastic processes, Contemporary Mathematics and Statistics, 2(2014), no. 1, 123-150.

9. R. F. ENGLE, C. W. J. GRANGER, Co-integration and error correction: Representation, estimation and testing, Econometrica, 55(1987), 251-276. 
10. C. W. J. GRANGER, Cointegrated variables and error correction models, UCSD Discussion paper 83-13a(1983).

11. U. GRENANDER, A prediction problem in game theory, Arkiv för Matematik, 3(1957), 371379.

12. J. Franke, Minimax robust prediction of discrete time series, Z. Wahrsch. Verw. Gebiete 68(1985), 337-364.

13. J. FrANKE, H. V. POOR, Minimax-robust filtering and finite-length robust predictors, Robust and Nonlinear Time Series Analysis. Lecture Notes in Statistics, Springer-Verlag, 26(1984), 87-126.

14. S.A. KASSAM, H. V. POOR, Robust techniques for signal processing: A survey, Proc. IEEE. 73(1985), 433-481.

15. A.N. Kolmogorov, Selected works by A. N. Kolmogorov. Vol. II: Probability theory and mathematical statistics. Ed. by A. N. Shiryayev. Mathematics and Its Applications. Soviet Series. 26. Dordrecht etc. Kluwer Academic Publishers, 1992.

16. M. M. LUZ, M. P. MOKLYACHUK, Interpolation of functionals of stochactic sequanses with stationary increments, Theor. Probability and Math. Statist., 87(2013), 117-133.

17. M. M. LUZ, M. P. MOKLYACHUK, Interpolation of functionals of stochastic sequences with stationary increments for observations with noise, Prykl. Stat., Aktuarna Finans. Mat., (2012), No. 2, 131-148.

18. M. M. LuZ, M. P. MOKLYACHUK, Minimax-robust filtering problem for stochastic sequence with stationary increments, Theor. Probability and Math. Statist., 89(2013), 115-129.

19. M. M. LuZ, M. P. MOKLYACHUK, Robust extrapolation problem for stochastic processes with stationary increments, Mathematics and Statistics, 2(2014), no. 2, 78-88

20. M. P. MOKLYACHUK, Minimax filtration of linear transformations of stationary sequences, Ukr. Math. J. , 43(1991), no. 1, 75-81.

21. M. P. MoklyachuK, Robust procedures in time series analysis, Theory Stoch. Process. 6(2000), no. 3-4, 127-147.

22. M. P. MOKLyACHUK, Game theory and convex optimization methods in robust estimation problems, Theory Stoch. Process. 7(2001), no. 1-2, 253-264.

23. M. P. MOKLYACHUK, Robust estimations of functionals of stochastic processes., Kyiv University, Kyiv, 2008.

24. M. P. MOKLYACHUK, Nonsmooth analysis and optimization, Kyiv University, Kyiv, 2008.

25. M. MOKLYACHUK, M. LUZ, Robust extrapolation problem for stochastic sequences with stationary increments, Contemporary Mathematics and Statistics, 1(2013), no. 3, 123-150.

26. M. MOKLYACHUK, A. MASYUTKA, Extrapolation of multidimensional stationary processes, Random Operators and Stochastic Equations, 14(2006), no. 3, 233-244.

27. M. MOKLYACHUK, A. MASYUTKA, Robust estimation problems for stochastic processes, Theory Stoch. Process. 12(2006), no. 3-4, 88-113.

28. M. MOKLyAChuK, A. MASYUTKA, Robust filtering of stochastic processes, Theory Stoch. Process. 13(2007), no. 1-2, 166-181.

29. M. MoklyachuK, A. MasyutKa, Minimax prediction problem for multidimensional stationary stochastic sequences, Theory Stoch. Process. 14(2008), no. 3-4, 89-103.

30. M. MOKLYACHUK, A. MASYUTKA, Minimax prediction problem for multidimensional stationary stochastic processes, Communications in Statistics - Theory and Methods. 40(2011), no. 19-20, 3700-3710

31. M. MoklyachuK, O. MASYUtKa, Minimax-robust estimation technique for stationary stochastic processes, LAP LAMBERT Academic Publishing, 2012.

32. M. S. PINSKER, A. M. YAGLOM, On linear extrapolaion of random processes with nth stationary incremens, Dokl. Akad. Nauk SSSR, 94(1954), no. 3, 385-388.

33. M. S. PINSKER, The theory of curves with nth stationary incremens in Hilber spaces, Izv. Akad. Nauk SSSR, Ser. Mat., 19(1955), no. 3, 319-344.

34. B. N. PSHENICHNYI, Necessary conditions of an extremum, "Nauka", Moskva, 1982.

35. Yu. A. Rozanov. Stationary stochastic processes. 2nd rev. ed, "Nauka". Moskva, 1990.

36. K. S. VASTOLA, H. V. POOR, An analysis of the effects of spectral uncertainty on Wiener filtering, Automatica, 28(1983), 319-344.

37. N. WIENER, Extrapolation, interpolation, and smoothing of stationary time series. With 
engineering applications, The M. I. T. Press, Massachusetts Institute of Technology, Cambridge, Mass., 1966.

38. A. M. YAGLOM, Correlation theory of stationary and related random functions. Vol. 1: Basic results, Springer Series in Statistics, Springer-Verlag, New York etc., 1987.

39. A. M. YAGLOM, Correlation theory of stationary and related random functions. Vol. 2: Suplementary notes and references, Springer Series in Statistics, Springer-Verlag, New York etc., 1987.

40. A. M. YAGLOM, Correlation theory of stationary and related random processes with stationary nth increments, Mat. Sbornik, 37(79)(1955), no. 1, 141-196.

41. A. M. YAGLOM, Some clases of random fields in n-dimentional space related with random stationary processes, Teor. Veroyatn. Primen., 11(1957), no. 3, 292-337. 